Wahringergurtel 18-20 A-1090 Vienna,Austria).

COMMENT. Plasma pipecolic acid determination may aid in diagnosis, and a persistent elevation may avoid premature pyridoxine withdrawal in infants with pyridoxine-dependent epilepsy (normal range, $0.7-2.6 \mathrm{mcmol} / \mathrm{L}$ ). The authors report an additional case of this syndrome in a 7 year-old child.

Pipecolic acid elevation in pyridoxine-dependent epilepsy may be caused by a deficiency of pyridoxal-dependent a-aminoadipic acid transaminase, a step in the degradation of lysine to acetoacetyl-CoA in the brain. Pipecolic acid also accumulates in peroxisomal disorders, such as Zellweger syndrome.

\title{
AUTOSOMAL DOMINANT PARTIAL EPILEPSIES
}

The clinical, electrophysiologic, and genetic characteristics of autosomal dominant partial epilepsy were studied in 71 patients and 33 non-epileptic at-risk family members in 19 European families followed at the Hopital Universitaire de Geneve, Switzerland, and centers in Strasbourg, Paris, Grenoble, Nice, Marseille, Rome, and Pisa. Families were subdivided into those with autosomal dominant nocturnal frontal lobe epilepsy (ADNFLE) $(n=8)$, familial temporal lobe epilepsy (7), and autosomal dominant partial epilepsy (4).

Familial partial epilepsies show great intrafamilial variability, and up to $30 \%$ may be resistant to antiepileptic medication. Some affected family members may have only EEG abnormalities, without clinical seizures, reflecting incomplete penetrance. Genetic studies found no mutation in the a4 and B2 nicotinic acethylcholine receptor subunits, but positive lod scores were obtained in 4 families with markers from the candidate region on chromosome 10q. (Picard F, Baulac S, Kahane $\mathrm{P}$ et al. Dominant partial epilepsies. A clinical, electrophysiological and genetic study of 19 European families. Brain June 2000;123:1247-1262). (Respond: Dr Fabienne Picard, EEG, Department of Neurology, Hopital Universitaire de Geneve, 24, rue Micheli-du-Crest, 1211 Geneva 14, Switzerland).

COMMENT. Familial partial epilepsies may originate in frontal, temporal, and other variable locations. Clinical and surface EEG findings may provide conflicting localizing evidence, and an overlap of partial epilepsy syndromes within families may occur, with genetic heterogeneity.

Frontal lobe origin of absence seizures is discussed by Pavone $\mathrm{A}$ and Niedermeyer E (Clin Electroencephalogr July 2000;31:153-156). The distinction between partial and absence seizures of frontal lobe origin is important from a therapeutic standpoint. Carbamazepine is the agent of choice for partial, and valproate for absence seizures.

\section{HHV-6, INFANTILE SPASMS, AND CEREBELLAR ASTOCYTOMA}

A case of human herpesvirus- 6 encephalitis, carditis, infantile spasms, and a subsequent cerebellar astrocytoma containing the HHV-6 genome, is reported from the University of Oulu, Finland. A 14-month-old girl presented with fever, hypotonia, and a diffuse urticarial exanthem. After admission, she developed encephalitis and status epilepticus, followed by myocarditis. MRI of the brain showed thin subdural effusions but no tumor. After 11 weeks from onset, convulsions changed to infantile spasms, resistant to vigabatrin and ACTH. A repeat $M R I$ at 11 months after the primary illness showed a cystic astrocytoma located near the vermis. Five months after surgical removal of the tumor, the patient has hypotonia, poor social communication, and daily infantile spasms. HHV-6 DNA was detected by PCR in the tumor tissue. (Rantala H, Mannonen L, 
Ahtiluoto S, Linnavuori $\mathrm{K}$ et al. Human herpesvirus- 6 associated encephalitis with subsequent infantile spasms and cerebellar astrocytoma. Dev Med Child Neurol June 2000;42:41 8-421). (Respond: Dr Heikki Rantala, Department of Paediatrics, University of Oulu, 90220 Oulu, Finland).

COMMENT. This is the first reported case of HHV-6 association with subsequent infantile spasms and pilocytic cerebellar astrocytoma. HHV-6 DNA found in the tumor cells may indicate a causative role for the virus or simply an invasion of the tumor by the virus. The absence of MRI evidence for a tumor at the onset of infection is in favor of the former explanation. Laboratory studies have demonstrated oncogenic properties of HHV-6 virus.

\section{MOVEMENT DISORDERS}

\section{VOLUMETRIC MRI IN TOURETTE SYNDROME: A GENDER EFFECT}

Subcortical volumetric MRIs were compared in 19 girls (aged 7 to 15 years) with Tourette syndrome (TS) and 21 controls. Eleven of the patients had TS only and 8 had TS with attention deficit hyperactivity disorder (TS + ADHD). TS-only patients had significantly smaller lateral ventricles compared with TS + ADHD patients and the control subjects. Putamen asymmetry index as a marker for TS, previously demonstrated in boys with TS, was present in girls with TS and also in control girls with no TS. (Zimmerman AM, Abrams MT, Giuliano JD, Denckla MB, Singer HS. Subcortical volumes in girls with Tourette syndrome. Support for a gender effect. Neurology June (2 of 2);54:2224-2229). (Reprints: Michael T Abrams, Kennedy Krieger Institute, Neuroimaging Laboratory, Suite 522, 707 North Broadway Street, Baltimore, MD 21205).

COMMENT. In contrast to previous studies involving mainly boys, abnormal lenticular asymmetry does not occur in girls with Tourette syndrome. Girls, with or without TS, exhibit the putamen asymmetry seen in boys. TS-only girls differed from TS + ADHD girls and controls in having significantly smaller lateral ventricles. Future investigations of TS should make distinctions of gender and patients with and without ADHD.

\section{TICS IN ASPERGER'S SYNDROME AND AUTISTIC DISORDER}

Twelve patients ( 8 male and 4 female; ages 3 to 32 years; 9 less than 16 years) with autistic spectrum disorder were evaluated in a Movement Disorders Clinic for tics, at the University of California, Irvine, CA. Six had Tourette syndrome, and eight were also diagnosed with Asperger's syndrome. Three TS patients had severe congenital sensory deficits; 2 with Leber's amaurosis and 1 with deafness. All had stereotypic motor behavior, including rocking, head banging, and hand waving. Sensory deprivation was thought to contribute to the stereotypic movement disorder. (Ringman JM, Jankovic J. Occurrence of tics in Asperger's syndrome and autistic disorder. I Child Neurol June 2000;15:394-400). (Dr Joseph Jankovic, Baylor College of Medicine, 6550 Fannin \#1801, Houston, TX 77030).

COMMENT. Asperger's syndrome should be considered in children of high verbal intelligence who do poorly in school, both academically and socially, and who exhibit speech and language disorders, tics, motor clumsiness, and stereotyped movements such as repetitive hand flapping. Asperger's syndrome may overlap or occur concurrently with Tourette syndrome (Nass R, Gutman R, 1997), pervasive developmental disorder, and ADHD (see Millichap JG. Attention 